\title{
A physical framework for implementing virtual models of intracranial pressure and cerebrospinal fluid dynamics in hydrocephalus shunt testing
}

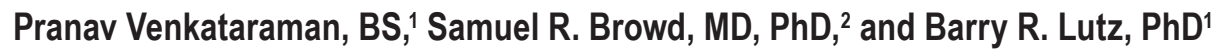 \\ 'Department of Bioengineering, University of Washington, Seattle; and 2Division of Pediatric Neurosurgery, Seattle Children's \\ Hospital, Seattle, Washington
}

\begin{abstract}
OBJECTIVE The surgical placement of a shunt designed to resolve the brain's impaired ability to drain excess CSF is one of the most common treatments for hydrocephalus. The use of a dynamic testing platform is an important part of shunt testing that can faithfully reproduce the physiological environment of the implanted shunts.

METHODS A simulation-based framework that serves as a proof of concept for enabling the application of virtual intracranial pressure (ICP) and CSF models to a physical shunt-testing system was engineered. This was achieved by designing hardware and software that enabled the application of dynamic model-driven inlet and outlet pressures to a shunt and the subsequent measurement of the resulting drainage rate.
\end{abstract}

RESULTS A set of common physiological scenarios was simulated, including oscillations in ICP due to respiratory and cardiac cycles, changes in baseline ICP due to changes in patient posture, and transient ICP spikes caused by activities such as exercise, coughing, sneezing, and the Valsalva maneuver. The behavior of the Strata valve under a few of these physiological conditions is also demonstrated.

CONCLUSIONS Testing shunts with dynamic ICP and CSF simulations can facilitate the optimization of shunts to be more failure resistant and better suited to patient physiology.

http://thejns.org/doi/abs/10.3171/2016.2.PEDS15478

KEY WORDS hydrocephalus; shunt; simulation system; intracranial pressure

$\mathrm{H}$ YDROCEPHALUS is characterized by the accumulation of excess cerebrospinal fluid (CSF) within the brain. Hydrocephalus affects individuals of all ages, especially newborn infants and older adults. ${ }^{11} \mathrm{Hy}-$ drocephalus is most commonly treated by surgically implanting a cerebral shunt, which is essentially a mechanical valve and tube that drains excess CSF from the ventricles to other body spaces such as the peritoneal cavity. Despite high failure rates, shunt design has changed little since these shunts were first used in the 1950s, mostly via valve designs intended to improve the fluid drainage characteristics. ${ }^{6,717,38}$ Advances have included the addition of antisiphon devices to reduce overdrainage, ${ }^{29}$ valves with externally adjustable pressure set points, and valves that regulate the flow rate rather than pressure. ${ }^{19}$ Valves are typically designed with static conditions in mind, but the dynamics of ICP and the CSF system can have unforeseen impacts on valve behavior (Supplementary Fig. S1). Electronic (i.e., smart) shunts are also being developed to address failure modes and allow sophisticated control of intracranial pressure (ICP). 1,2,30,35-37 These smart shunts are designed to sense and maintain ICP within acceptable levels through a control algorithm that needs to respond appropriately to fast and slow ICP changes while saving power for long-term battery operation. Dynamic testing systems play an essential role in the testing and development of conventional mechanical shunts and emerging smart shunts. 
Shunt drainage is affected by dynamic conditions such as posture changes and fluctuations induced by exercise, patient movement, and complex physiological cycles in CSF production and cerebral blood flow. For example, ICP constantly oscillates because of the influence of respiratory, cardiac, and other endogenous pressure waves in the brain. Oscillating ICP produces a pumping effect in many differential pressure valves that leads to an increase in the amount of drained CSF.,15 This effect may be exacerbated with strenuous exercise and other circumstances with elevated respiratory and heart rates. Lundberg B waves, which produce high-amplitude fluctuations in cerebral blood flow (and consequently ICP) because of alternating dilation and constriction of the cerebral vascular bed, ${ }^{12-15,23}$ are another example of this phenomenon. The pumping effects driven by these fluctuations cause overdrainage, which leads to a gradual drop in ICP that presents as morning headaches.

Several benchtop rigs have been designed over the years to test shunts. Aschoff et al. ${ }^{3}$ documented setups designed to test the resistance and pressure-dependent flow rates of shunt valves and used physical changes in the system to model physiological conditions such as sinusoidal waves, impulse waves, and siphoning. Czosnyka et al.${ }^{15}$ built a rig with an electronic pulse-pressure generator for producing ICP waves, and they tested for the effects of siphoning, reflux, and impulse waves on shunt behavior. Elixmann et al. ${ }^{19,27}$ designed a benchtop representation of the CSF system with a physical model of brain compliance that was based on a sponge in a fluid compartment. On the other hand, many completely virtual models of ICP and CSF dynamics have been developed, including the 6-compartment model proposed by Ko et al., ${ }^{26}$ but these dynamics are excluded from typical shunt-testing systems.

Shunt-testing systems that use physical models require the hardware to be changed to simulate different physiological conditions (e.g., brain tissue compliance) and, thus, do not allow for the "on-the-fly" changes needed to simulate the physiological changes that would occur in real time within a patient (e.g., instantaneous variations in brain compliance with changing ICP). Our ultimate goal is to transcend these limitations by creating a smart system that is capable of actively responding to shunt performance under real-time patient scenarios (e.g., minute-tominute variations in physiology in response to movements and posture changes) and using shunt responses to generate a new set of simulated testing conditions. Here, we present an essential step toward this goal by developing a flexible hardware-software interface that allows common dynamic conditions to be manipulated arbitrarily from a computer without changes to the hardware itself. Figure 1 shows the conceptual framework for a shunt-testing system based on a virtual model. At any instant, a shunt is subject to an applied ICP at the inlet and either abdominal or siphoning pressure at the outlet, and the flow through the shunt is measured. By creating a cycle with a virtual model, the measured drainage rate could be used to calculate a new condition based on a patient brain model or patient scenario (e.g., when the patient stands up) with the new condition being physically applied to the shunt, and so on. Here, we present a framework that is configured to test shunts affected by respiratory and cardiac ICP waves (with tunable frequencies and amplitudes), ICP amplitude elevations that occur with low brain compliance, posturedependent ICP changes, impulse waves, noise, abdominal pressure effects, and siphoning.

\section{Methods}

The goal of the hardware was to apply desired pressures to the inlet and outlet of the shunt and measure the resulting flow rate. Dynamic pressures were classified as 1) long-timescale changes (e.g., patient position, drainage responses) that were applied by the pressure/vacuum sources and 2) short-timescale changes (e.g., noise, impulses, pulsations) that were applied by the speakers. The testing rig in Fig. 2 consisted of 2 tanks representing the cephalic and abdominal spaces. Each tank consisted of a closed Plexiglas cylinder fitted with a speaker on the top (Dayton Audio DA115-8 4 Aluminum Cone Woofer),

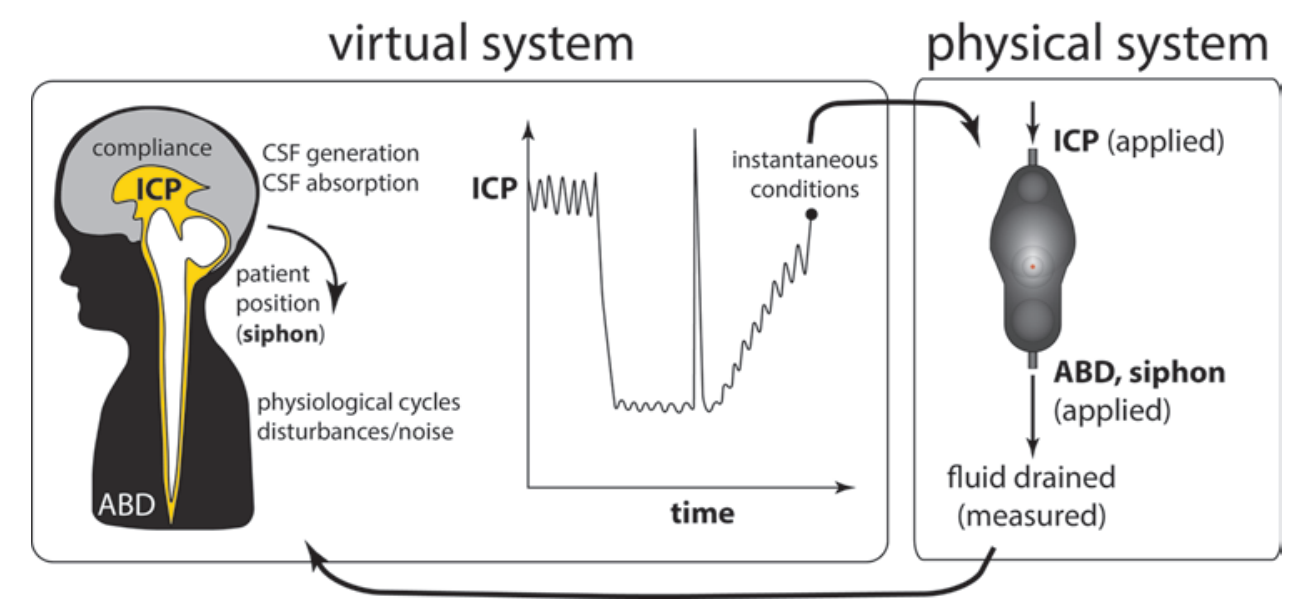

FIG. 1. Flowchart demonstrating the closed-loop, feedback-based interaction between the shunt and simulation system. The virtual model applies input pressures onto the physical system to which the shunt responds. Shunt response is fed back into the virtual system through pressure and level sensing, which then outputs updated pressure and volume control signals back into the physical system. $A B D=$ abdomen. Figure is available in color online only. 


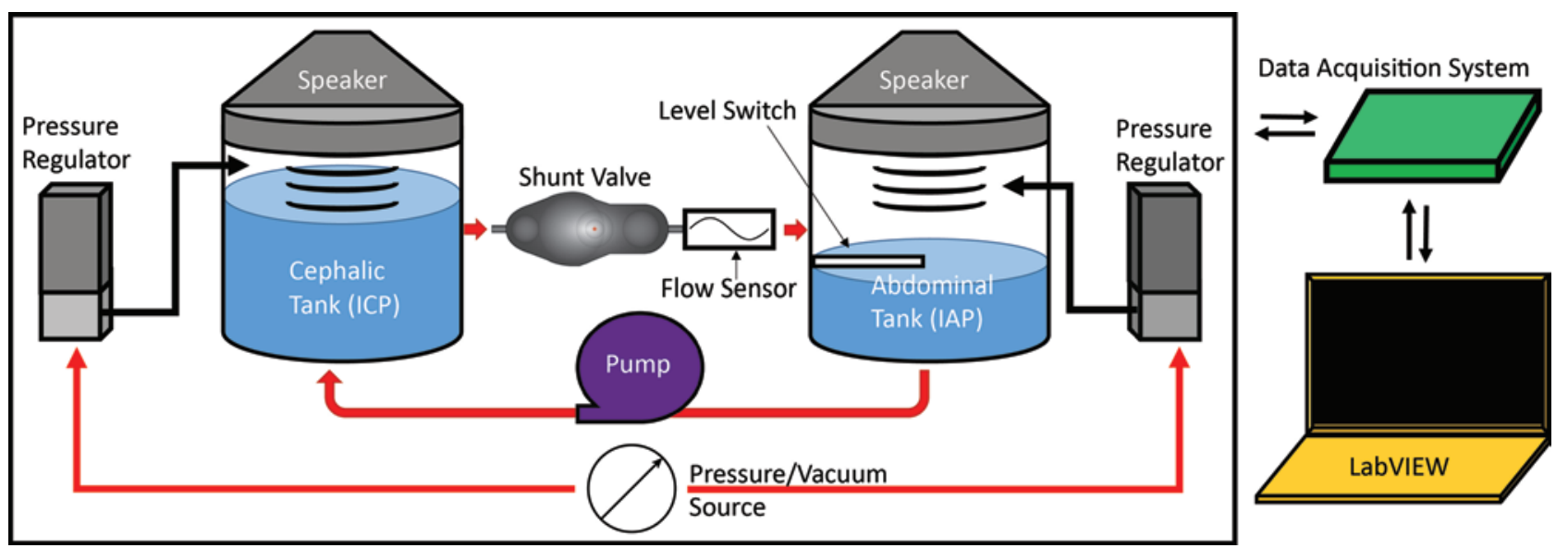

FIG. 2. Complete schematic of the testing system. The shunt drained fluid from the cephalic tank to the abdominal tank. To maintain closed-loop volume control, a pump (controlled by the feedback signal from the computer) was used to replace the drained fluid. Tank pressures were maintained around set points using pressure regulators that are controlled via the virtual system. The speakers controlled by the virtual system were used to apply pressure oscillations upstream and/or downstream of the shunt. Figure is available in color online only.

which was used to produce oscillations and other short timescale disturbances. Inlet pressure (i.e., ICP) and outlet pressure (i.e., abdominal) were defined as the air pressures above the water in each cylinder and were measured using electronic pressure sensors (HCLA series; SensorTechnics).

The average ICP levels were controlled in the tanks using electronic airflow regulators for low-pressure applications (Kelly Pneumatics, Inc.). These regulators (which were fitted with internal pressure sensors) were connected to house air and vacuum sources and used to set and maintain the desired baseline ICP. The response time of the regulators was tuned to prevent them from reacting to short-timescale transients, ensuring that regulator control did not interfere with the simulating ICP waves, noise, and impulse spikes within the tanks. A level switch (Omega Engineering LVK-91) was fitted to the abdominal tank to monitor its water level, and a peristaltic pump was used to pump water (i.e., a mock CSF) from the abdominal to the cephalic tank to counteract shunt drainage and maintain constant water levels in the tanks. When testing the shunts, a flow sensor (Sensirion ASL 1600) was added to the distal catheter to measure the flow rate through the shunt. Each tank was fitted with a port to allow connection to the shunt tubing.

All electronic devices were connected to a National Instruments USB-6259 Data Acquisition System for controlling the system and receiving data. The data acquisition system was controlled from a computer using National Instruments LabVIEW Software. A LabVIEW control panel was programmed to adjust the pressure set point in both tanks through the regulators and record the pressure in both tanks. A control loop connecting the pump and level switch was set up to maintain the tank water level (Supplementary Fig. S2). When the water in the abdominal tank rose to the switch point (because of shunt drainage), the level switch would activate the pump. Speakers were used to simulate short timescale ICP spikes, as well as the longer timescale oscillations from the respiratory, cardiac, and $\mathrm{C}$ waves. Speaker controls were used to adjust the frequency and amplitude of the ICP waves. The simulation results were recorded in LabVIEW, written to an Microsoft Excel file, and plotted. The flow sensor was operated independently of LabVIEW using Sensirion measurement software.

A shunt-testing experiment was performed using the simulation system by connecting a Strata valve shunt to the system. The valve was obtained for research purposes after it was opened for a planned surgery but not used. Before initiating the experiment, the valve was first rid of air bubbles, and a finite pressure (i.e., ICP) was applied to the cephalic tank. Flow through the distal shunt catheter was measured and recorded using the electronic flow sensor, and fluid was recycled into the cephalic tank from the abdominal tank using the peristaltic pump. ICP and CSF flow measurements through the experiment's time course were jointly plotted in Microsoft Excel. The experiment involved a single run of measurements under 2 conditions: oscillating ICP and nonoscillating ICP.

\section{Results}

To demonstrate the system, we focused on 5 dynamic conditions: 1) basal ICP oscillations (respiratory, cardiac, and $\mathrm{C}$ waves); 2) short-timescale disturbances (rapid, transient, and irregular spikes); 3) ICP amplitude dependence on baseline pressure; 4) posture-dependent ICP changes; and 5) abdominal pressure and shunt-siphoning effects. The ICP parameters reported in the literature (Table 1) were used to define the test conditions.

\section{Respiratory, Cardiac, and C Waves}

Slow- to medium-timescale waves of ICP typically manifest as respiratory, cardiac, and nonpathological slow waves, the most important of which are $\mathrm{C}$ waves. ${ }^{13,28} \mathrm{Re}-$ spiratory waves are caused by the breathing cycle and affect ICP by changing intrathoracic pressure and, consequently, systemic venous return and blood pressure. ${ }^{25}$ 
TABLE 1. Index of physiologically important ICP parameters and their magnitudes

\begin{tabular}{lll}
\hline \multicolumn{1}{c}{ Authors \& Year } & \multicolumn{1}{c}{ Parameter } & \multicolumn{1}{c}{ Magnitude } \\
\hline Sakka et al., 2011 & Normal ICP (recumbent) & $15 \mathrm{~cm} \mathrm{H}_{2} \mathrm{O}$ \\
\hline $\begin{array}{l}\text { Chapman et al., 1990; } \\
\text { Foltz et al., } 1994\end{array}$ & Normal ICP (upright) & $\sim-6.6-0 \mathrm{~cm} \mathrm{H}_{2} \mathrm{O}$ \\
\hline Juul et al., 2000 & Hypertension threshold & $26-32.5 \mathrm{~cm} \mathrm{H}_{2} \mathrm{O}$ \\
\hline Lemaire et al., 2002 & C-wave amplitude range & $0-27.2 \mathrm{~cm} \mathrm{H}_{2} \mathrm{O}$ \\
\hline Dunn, 2002 & C-wave frequency range & $4-8$ waves $/ \mathrm{min}^{\prime}$ \\
\hline Czosnyka et al., 2004 & Respiratory amplitude & $<1 \mathrm{~cm} \mathrm{H}_{2} \mathrm{O}$ \\
\hline Daley et al., 1982 & Cardiac amplitude & $0.78-9.36 \mathrm{~cm} \mathrm{H}_{2} \mathrm{O}$ \\
\hline
\end{tabular}

Cardiac ICP waves have a much higher frequency that corresponds to the heart rate. Each systolic contraction leads to an increase in arterial pressure that produces a transient ICP increase ${ }^{4,26} \mathrm{C}$ waves, on the other hand, are low-frequency oscillations that are an intrinsic property of
ICP with causes that are not yet fully understood. ${ }^{23}$ Figure 3 shows simulated respiratory, cardiac, and $\mathrm{C}$ waves. In our system, ICP waves were specified by their virtual frequency and amplitude, and the resulting combined waveform was used to drive the speakers.

\section{Rapid, Transient, and Irregular Spikes in ICP}

ICP can also be altered by spikes in pressure caused by activities such as exercise, coughing, sneezing, and Valsalva maneuvers. Brimioulle et al. ${ }^{5}$ measured ICP spikes in physiotherapy patients who were asked to perform hip adduction exercises, and Coté et al..$^{10}$ simulated the ICP impulse response associated with random transient events such as coughing and sneezing. In our system, short-timescale, high-amplitude changes were produced in the tanks using the speakers (Fig. 4). The amplitude, frequency, and duty cycle can be adjusted to modify the strength, rate, and duration of each ICP spike to model different types of exercises, as well as coughing, sneezing, and valsalva.
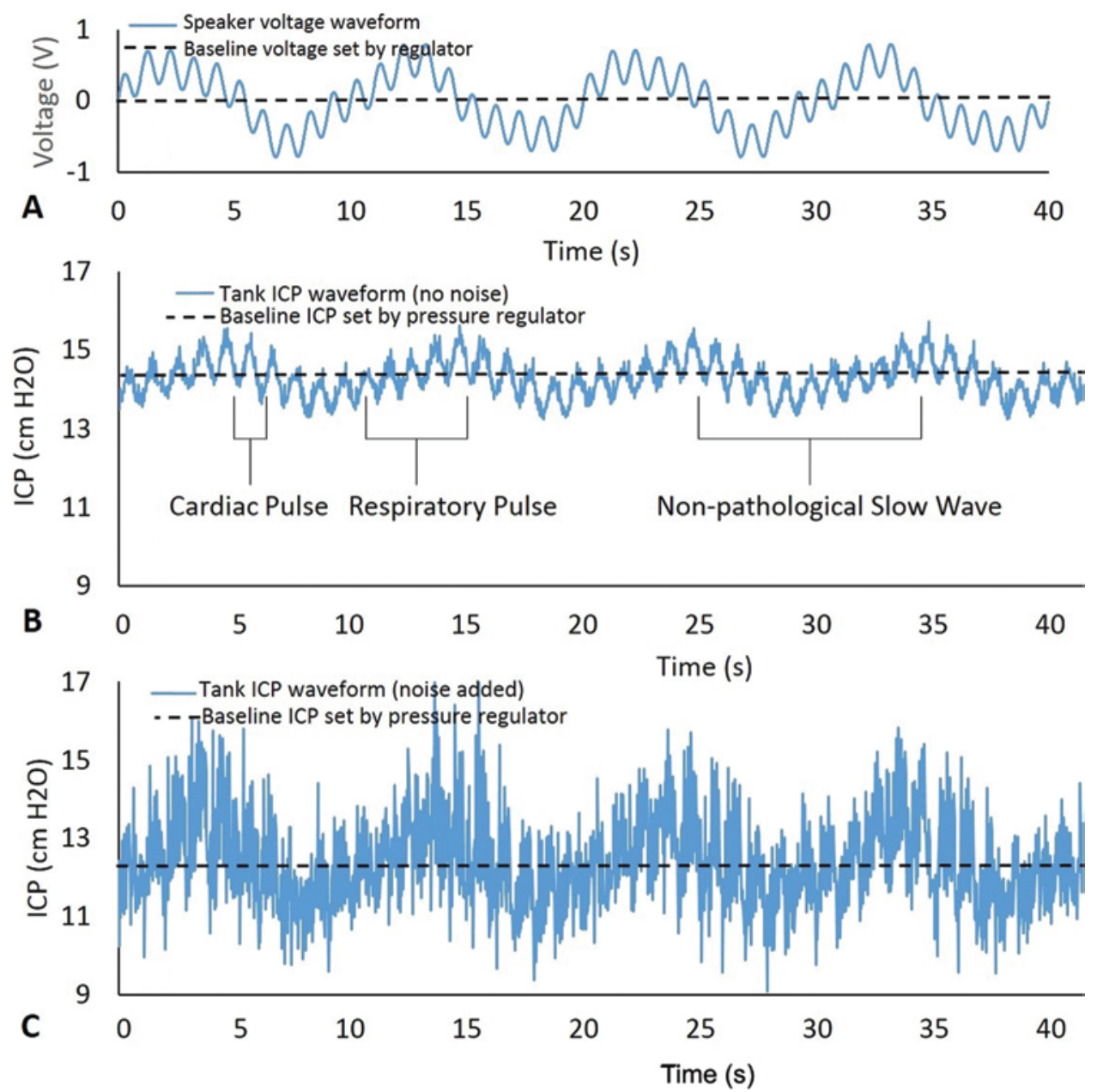

FIG. 3. Inducing basal oscillations in ICP by inputting modified sine voltage waves into the speaker. The panels show time-dependent ICP waveforms representing a healthy resting adult in the supine position. The heart rate is 60 beats/minute, and the respiratory rate is 15 breaths/minute. The major contributors to the waveform are cardiac, respiratory, and nonpathological slow waves. Of these, the respiratory contribution is the smallest at an amplitude $<1 \mathrm{~cm} \mathrm{H}_{2} \mathrm{O}$. A: Waveform of voltage input into a speaker coupled to the cephalic tank. B: The pressure waveform measured in the cephalic tank. C: The pressure waveform measured in the cephalic tank with random high-frequency noise added. Figure is available in color online only. 

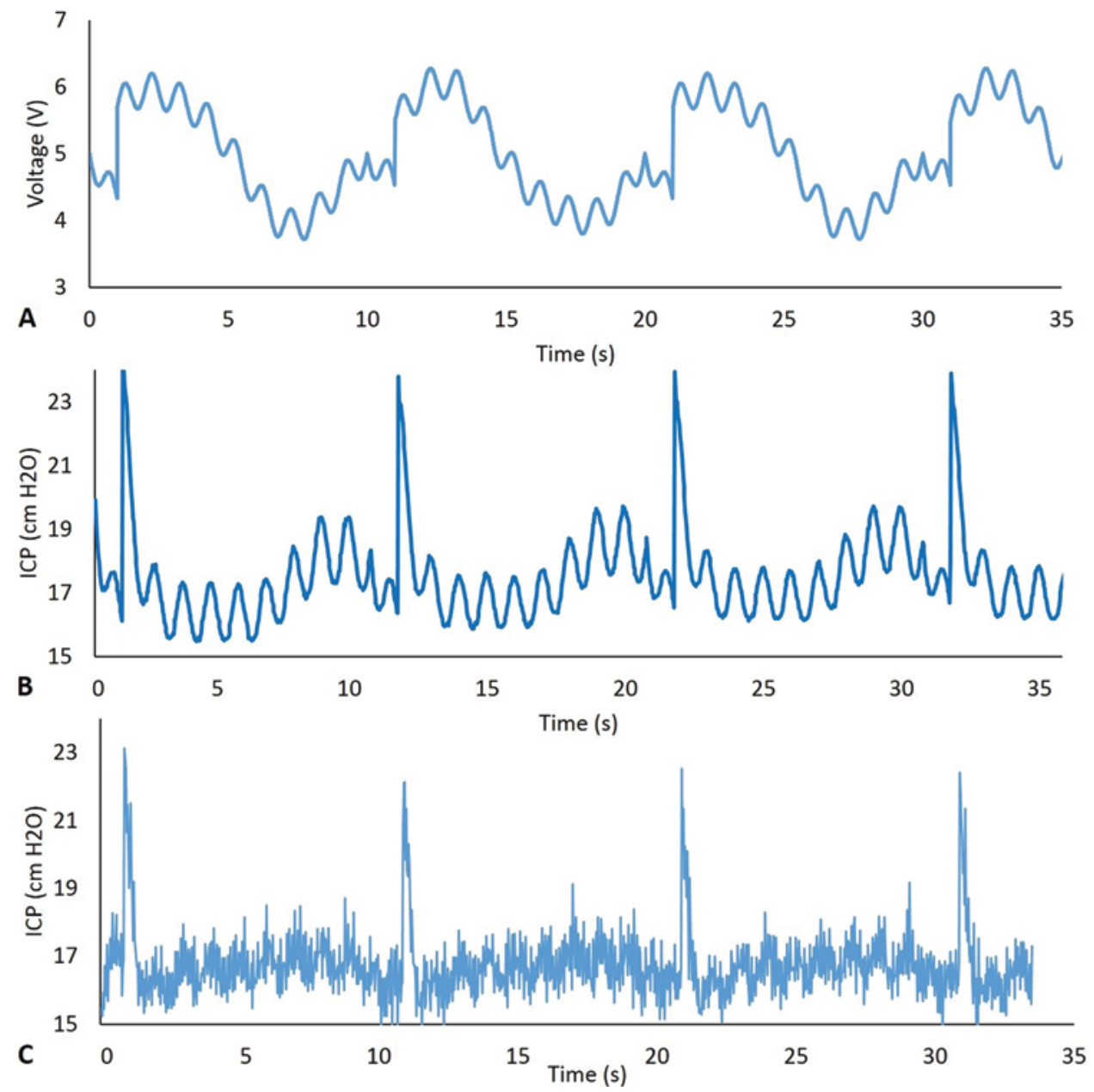

FIG. 4. Inducing high-amplitude spikes in ICP by inputting high-voltage and low duty-cycle pulse waves into the speaker. Transient high amplitude spikes are commonly caused by coughing, sneezing, and exercise and can result in overdrainage through the shunt. A: Waveform of the voltage input into the speaker coupled to the cephalic tank. B: The pressure waveform measured in the cephalic tank. C: The pressure waveform measured in the cephalic tank with random high-frequency noise added. Figure is available in color online only.

\section{Effect of Baseline ICP on the Amplitudes of the Waves of All Timescales}

As ICP increases, brain compliance and the ability to maintain a constant net intracranial volume decrease. ${ }^{34}$ This mechanism eventually leads to an exponential increase in ICP with relatively small changes in intracranial volume. ${ }^{40}$ This phenomenon is also observed with ICP waves: as baseline ICP increases, the corresponding decrease in brain compliance leads to higher wave amplitudes than what is observed under physiologically normal ICP. ${ }^{15,40}$ Figure 5 shows the comparison of amplitude dependence on baseline ICP as presented by Keong et al. ${ }^{24}$ with those simulated in our physical system. As baseline ICP increases, the amplitudes of the simulated respiratory, cardiac, and $\mathrm{C}$ waves also increase. Increases in amplitude were simulated through control of the voltage output to the speaker. ICP baseline was established at the set point by the pressure regulators, and the amplitude of the voltage output to the speaker was increased to simulate the larger magnitude due to reduced compliance.

\section{Posture-Dependent CSF Drainage}

Switching from the recumbent position to the upright position leads to a decrease in ICP. ${ }^{23,24,33}$ This is caused by a reduced venous return and pooling of blood in the lower extremities. The decrease is gradually compensated for by endogenous blood pressure control mechanisms and CSF production by the choroid plexus, which leads to progressive ventricular filling. Figure 6 shows a comparison of data from the literature with the simulated conditions in our system. Figure 6A from Chapman et al. ${ }^{8}$ demonstrates a decrease in ICP with an increase in the angle of posture from the horizontal. Figure 6B shows an initial decrease in ICP when sitting upright that eventually increases because of the filling phase, ${ }^{31}$ and Fig. $6 \mathrm{C}$ demonstrates this effect in our physical system. Posture-dependent drainage was simulated by tuning the starting and ending ICP baseline values and the timing of ICP recovery in the upright state due to compensatory mechanisms such as filling. Both normal and pathological conditions are demonstrated. Pathological conditions were modeled with higher than 

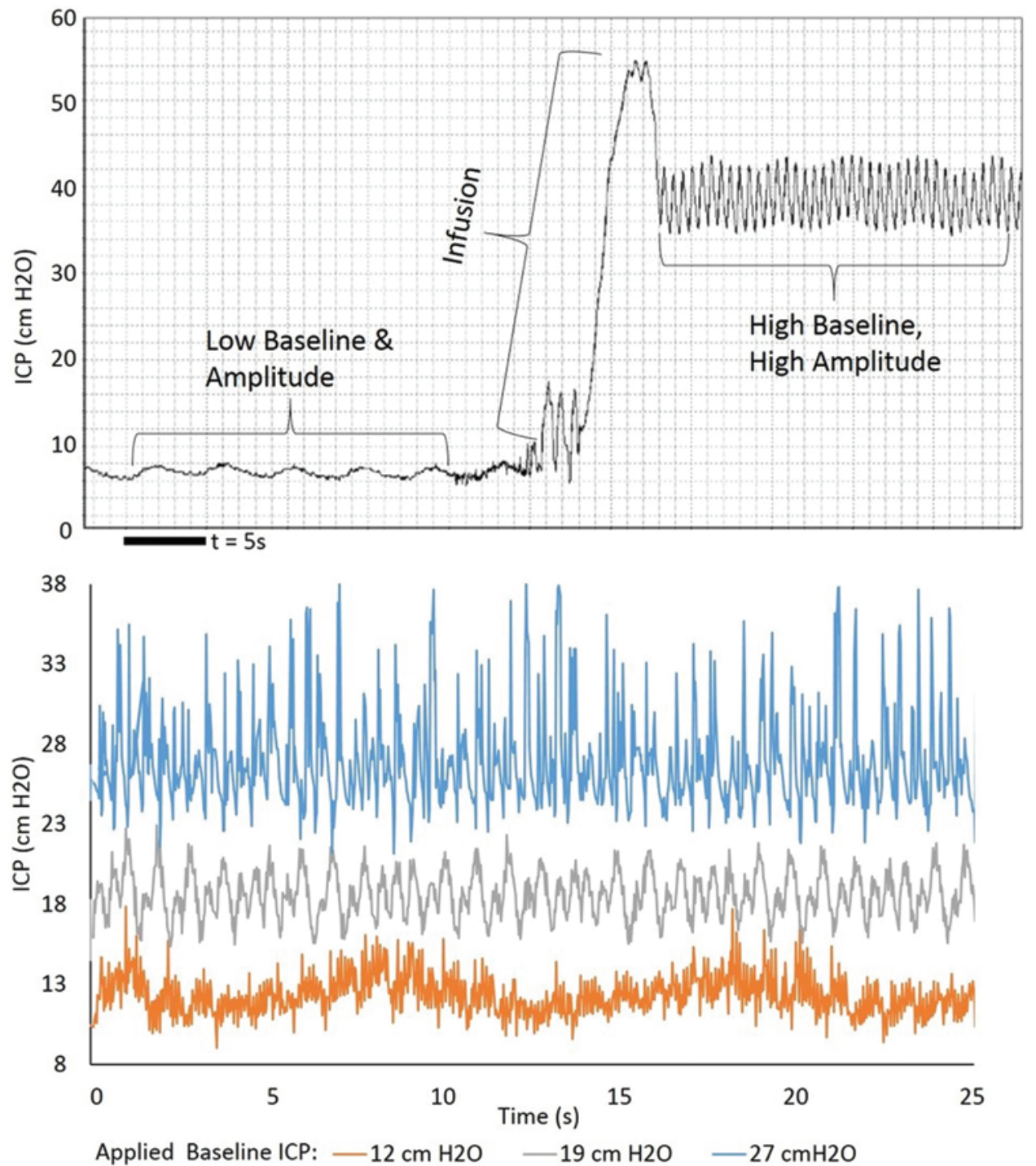

FIG. 5. Reproducing an increase in ICP amplitude due to an increase in baseline ICP. Intracranial hypertension reduces the compensatory reserve (compliance) of the brain, leading to high ICP amplitudes for pulsatile increases in intraventricular volume. At high baseline pressures, this effect is offset by increased shunt drainage. Upper: The literature results of a CSF infusion test performed in a patient. The increase in baseline ICP caused by infusion also leads to increased waveform amplitudes. Figure was published in Keong N, Czosnyka M, Czosnyka Z, Pickard JD: Clinical evaluation of adult hydrocephalus, in Winn H (ed): Youman's Neurological Surgery, ed 6, pp 494-514. Philadelphia: Elsevier, 2011, copyright Elsevier. Published with permission. Lower: Data from the simulation system illustrating elevated ICP amplitudes at high baseline ICP. Figure is available in color online only.

normal upright and recumbent ICPs, higher maximum upright pressure, and a faster than normal rise in filling pressure, the latter two of which are characteristics of reduced brain compliance.

\section{Abdominal Effects and Shunt Siphoning}

Intraabdominal pressures (IAPs) can affect shunt function, and IAP simulations can be a useful way of testing shunt reflux. In our system, the IAP effects are tested by changing the set point pressure in the abdominal/peritoneal tank that is connected to the shunt outlet. To test reflux, a high positive pressure is applied inside the tank, and the magnitude and direction of the flow through the shunt is measured in response to these changes in order to assess the degree of reflux.

\section{Shunt-Testing Experiments}

The Medtronic Strata valve was first tested with basal ICP oscillations (respiratory and cardiac cycles) and then spikes. The results of our testing are shown in Fig. 7. To test for this phenomenon, the average measured ICP and flow through the shunt with oscillating pressure is compared with the same parameters when basal oscillations (respiratory, cardiac, and $\mathrm{C}$ waves) are absent. The mean ICPs in both scenarios were tuned to be virtually the same value: $13.6 \pm 0.1 \mathrm{~cm} \mathrm{H}_{2} \mathrm{O}$ without oscillations and $13.8 \pm$ 
$0.8 \mathrm{~cm} \mathrm{H}_{2} \mathrm{O}$ with oscillations. Interestingly, the flow rates, as measured by the flow sensor, were significantly different for these 2 scenarios. A rate of $462 \pm 6 \mu \mathrm{l} /$ minute was measured without oscillations, while a rate of $494 \pm 15 \mu \mathrm{l} /$ minute was measured with basal oscillations.

\section{Discussion}

An important limitation regarding the ICP parameters provided in Table 1 is that they are primarily obtained from experimental measurements made in adults and therefore may or may not be representative of a pediatric population. Adding to this limitation is the paucity of information in the literature on certain characteristics of intrinsic ICP waves (e.g., C-wave amplitude and frequency), with the sources listed here being among the very few that provide concrete values for these parameters (the values for pediatric patients are even more scarce). Nevertheless, our goal was to design a flexible test bed that would appropriately accommodate and adapt to a variety of physiological parameters. As such, the parameters that we provide here merely serve illustrative purposes meant to demonstrate the test bed's working capability.
With respect to simulating respiratory, cardiac, and C waves, we wanted to make sure that our system can incorporate a good deal of physiological variability in our simulations. To this end, the frequency and amplitude of the respiratory and cardiac waves were made tunable through LabVIEW's front-panel controls (Supplementary Figs. S2 and S3) to virtually model variations in the heart rate, breathing rate, and blood pressure that can then be physically simulated by the testing hardware. Testing shunts through simulations of physiological variations within the CSF system, which we have demonstrated in our setup, will play an important role in achieving the following treatment goals: 1) optimized shunt function to suit each patient's personalized needs, and 2) flexible and adaptable shunt responses that are well suited to intrapatient physiological variability.

With regard to simulating spikes in ICP, the amplitudes of these spikes will be lower in a patient with a properly functioning shunt (as opposed to a patient without a shunt) because of compensation by shunt drainage. ${ }^{15}$ In addition, it is important to simulate fast-timescale changes in ICP in order to enable the optimization of shunts and prevent overdrainage. Simulation of fast-timescale changes will be
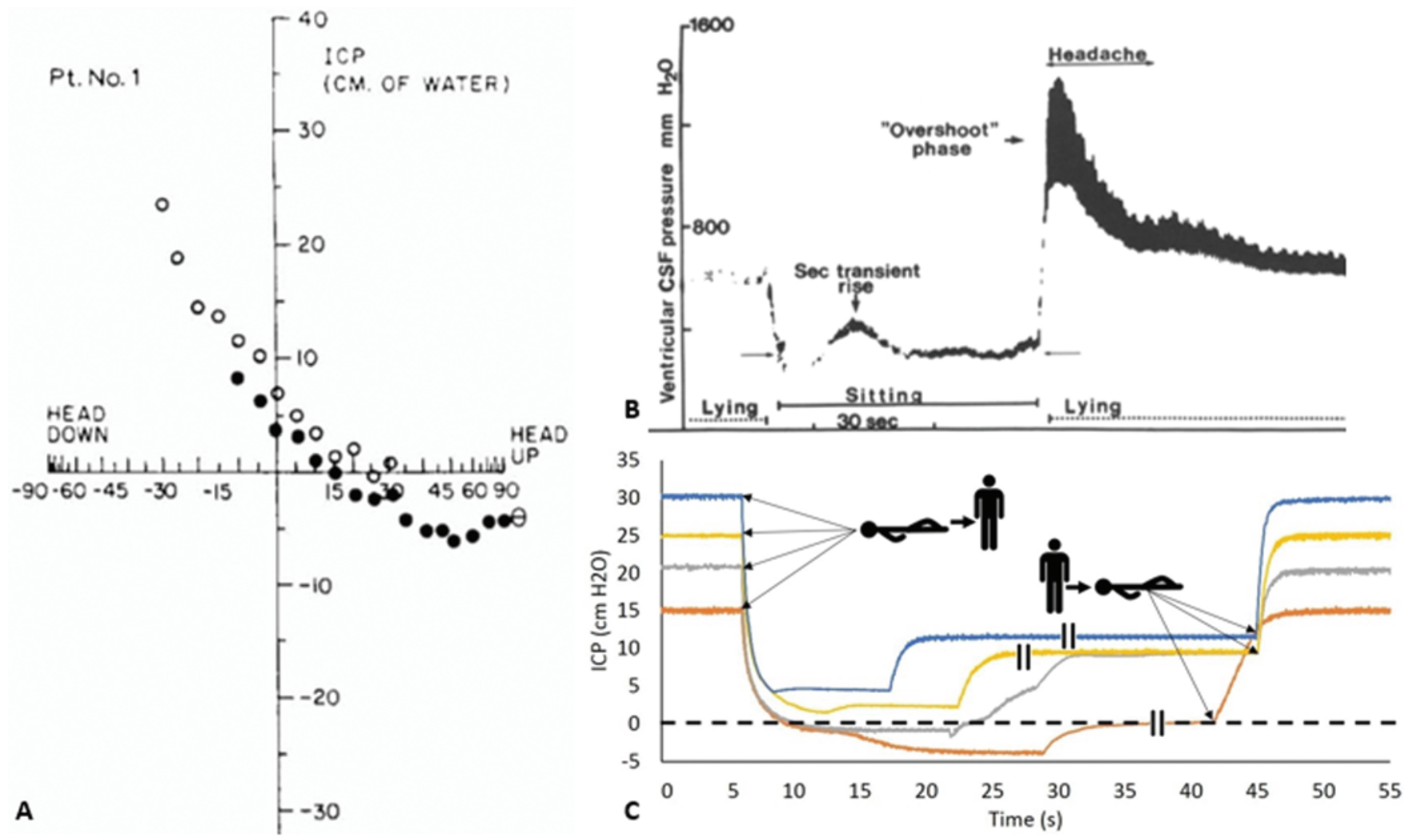

FIG. 6. Posture-dependent changes in ICP. A: The literature results demonstrating ICP levels at the sine of various angles of depression or elevation, with a drop in ICP associated with the transition to upright posture. Reproduced with permission from Chapman PH, Cosman ER, Arnold MA: The relationship between ventricular fluid pressure and body position in normal subjects and subjects with shunts: a telemetric study. Neurosurgery 26:181-189, 1990. B: ICP shift produced in a patient with aqueductal occlusion by switching from lying down to sitting up. After sitting up, the initial ICP drop is followed by a slow rise due to ventricular filling. Reproduced with permission from Magnaes B: J Neurosurg 44:687-697, 1976. C: Data from the simulation system illustrating position-dependent ICP changes under normal and pathological conditions. In patients with aqueductal occlusion, baseline ICP is elevated and the rate of ICP increase is higher during the filling phase of the upright position. To align the time of the transition points for all traces, a portion of the steady-state trace in the upright position is not shown for 3 of the 4 traces. Figure is available in color online only. 


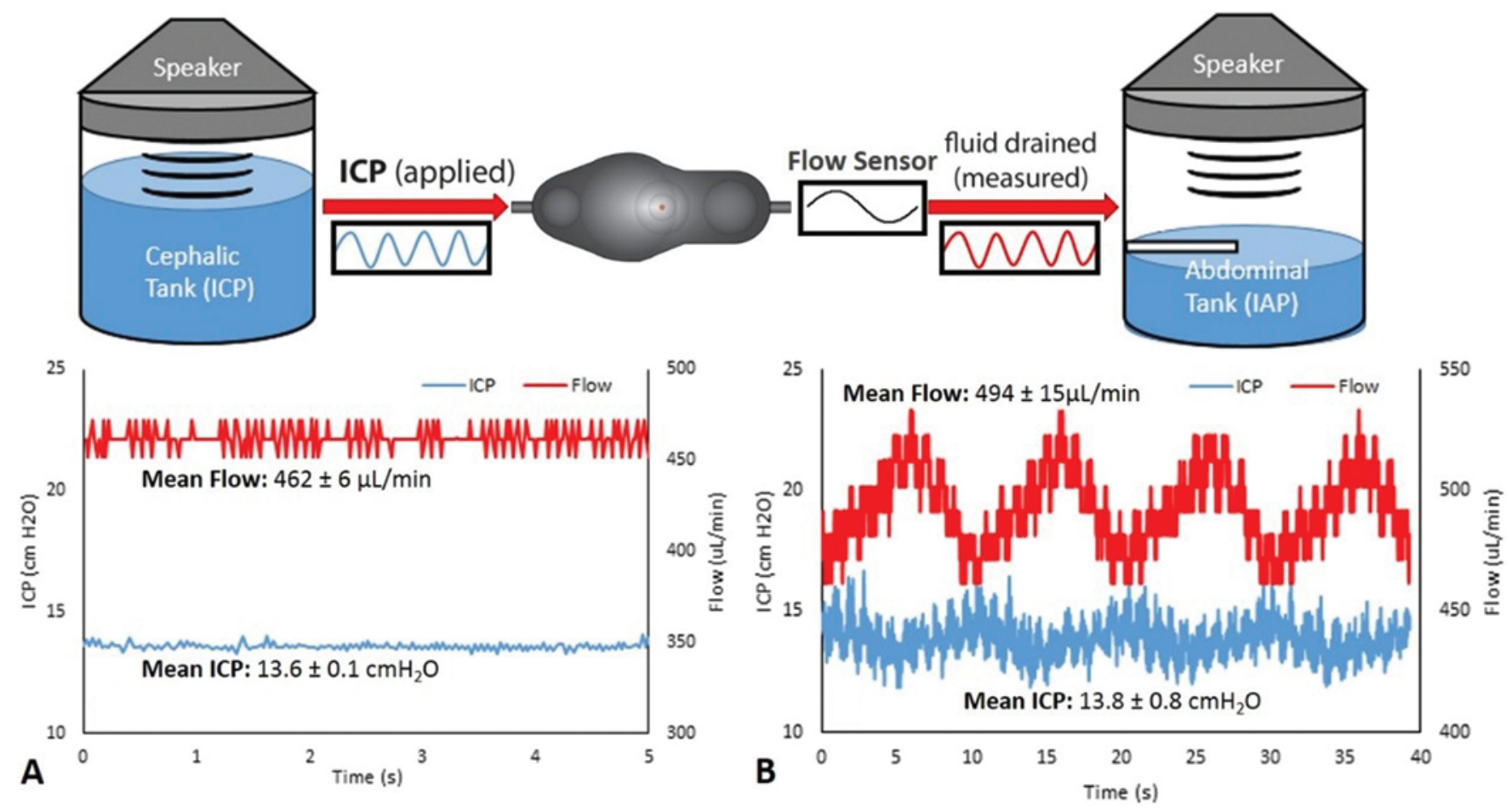

FIG. 7. Baseline ICP applied to the system in the cephalic tank and the resulting flow rate through the shunt as measured by the flow sensor are plotted $(A)$ and compared with the same parameters under basally oscillatory ICP (respiratory, cardiac, and $C$ waves) (B). Except for measurement noise (which is present in both testing scenarios), the ICP trajectory plotted in panel A does not contain any virtually imposed oscillations. There is no significant difference in the average applied ICP( $\mathrm{t})$ across both conditions (13.6 $\pm 0.1 \mathrm{~cm} \mathrm{H}_{2} \mathrm{O}[\mathrm{A}]$ vs $\left.13.8 \mathrm{~cm} \mathrm{H}_{2} \mathrm{O} \pm 0.8 \mathrm{~cm} \mathrm{H}_{2} \mathrm{O}[\mathrm{B}]\right)$. There is a significant difference in the average applied flow across both conditions (462 $\pm 6 \mu \mathrm{l} / \mathrm{min}[\mathrm{A}]$ vs $494 \pm 15 \mu \mathrm{l} / \mathrm{min}[\mathrm{B}]$ ), suggesting a pumping effect under oscillating conditions. Figure is available in color online only.

especially important in testing smart shunts, which must be programmed to appropriately interpret discrete pressure measurements (e.g., a measurement made during a short spike should not be interpreted as high ICP that requires drainage).

In our simulation for testing the effect of baseline ICP on the amplitudes of ICP oscillations, one particular limitation we encountered was the inability to increase the speakers' displacement indefinitely. At a certain baseline air pressure in the tank (i.e., baseline ICP), the opposing force was too large for the speaker, thereby limiting the amplitude of the speaker's pressure waves. However, this did not detract from our ability to reliably test a working shunt because unless the shunt has severely malfunctioned or been completely obstructed, excessively high ICPs have a low chance of occurrence..$^{15}$ Thus, the system was able to produce the magnitude of pressure oscillations expected for a typical range of ICP.

When it comes to testing patient positioning on CSF dynamics, the effects of patient posture on the shunts need to be tested because of the importance of siphoning and overdrainage in shunt design. This simulation can also be used to test the response times of the smart shunts to orthostatic ICP changes and can ultimately facilitate the selection of the control parameters that lead to optimal drainage control and power draw in smart shunts. Furthermore, siphoning by shunts is a major cause of overdrainage in the upright position. ${ }^{21}$ Siphoning pressure, which is directly proportional to the distance between the ventricles and the peritoneal cavity, is high in both pediatric and adult patients (although much higher in adults). Both physiologically and in our model, elevated IAP contributes to a positive outlet pressure that can lead to reflux, while siphoning acts as a vacuum that can lead to overdrainage. Oscillatory wave components can also be imposed on these pressures through our virtual model.

In showing a differential flow rate across valves with identical mean ICPs under oscillating and non-oscillating conditions, the results of the shunt testing experiment effectively demonstrate a pumping effect with net fluid loss at a given baseline pressure when oscillations are present. This phenomenon is observed because oscillations in ICP lead to increased shunt drainage over time compared with what was observed at baseline ICP, leading to the conclusion that the 1-way valve of the shunt becomes a pump under oscillating pressures.

\section{Conclusions}

Our main purpose here was to establish a proof-ofconcept system to allow virtual models of ICP and CSF dynamics to be integrated into shunt-testing procedures. By simulating a variety of physiological variations in ICP and using simulations to test shunt drainage, we have 
demonstrated that our testing framework can be used as a foundation upon which more complex virtual models can be constructed to test for more sophisticated performance factors.

A useful implementation of this system would incorporate an automated feedback model to bridge the physical and virtual systems. We envision this as an interaction in which the physical model receives continuous inlet (i.e., ICP) and outlet (i.e., IAP or siphoning) pressures as inputs and the measured volume of drained CSF is fed back to the virtual model, which is programmed to generate a new set of instantaneous inputs (see Fig. 2). The virtual model can be expressed as any algorithm that the researcher would like to simulate, including the dynamics of the CSF system and patient scenarios. This type of approach is useful for modeling changes in brain compliance and intracranial volume (ICV[t]) and their relationship to ICP changes. Unlike physical compliance models, a dynamic feedback scheme allows for modeling compliance as a time-dependent relationship between $\triangle \mathrm{ICP}$ (input) and $\triangle \mathrm{ICV}(\mathrm{t})$ (output) based on the brain compliance models that represent different patient populations and scenarios.

For testing smart shunts, the figure of merit (FOM) is a quantitative assessment of electronic shunt performance and power consumption that is calculated using algorithms that can be incorporated into the testing system. ${ }^{35}$ FOM algorithms can be run alongside the electronic simulations of the testing system in order to assess the smart shunt's response to various physiological ICP fluctuations. As such, FOMs are a potentially useful way to model the quantitative relationship between power consumption and physiological conditions and will perhaps help establish an optimal balance between efficiency and efficacy in smart shunts.

\section{Acknowledgments}

We thank Dr. Paul Yager, professor at the University of Washington Department of Bioengineering, for granting us access to his laboratory facilities and Peter Kauffman for supplying and assisting with the electronics.

\section{References}

1. Al-Zubi N, Al-Kharabsheh A, Momani L, Al-Nuaimy W: Intelligent shunt agent for gradual shunt removal. Conf Proc IEEE Eng Med Biol Soc 2010:430-433, 2010

2. Alkharabsheh AR, Momani L, Al-Zu'bi N, Al-Nuaimy W: An expert system for hydrocephalus patient feedback. Conf Proc IEEE Eng Med Biol Soc 2010:1166-1169, 2010

3. Aschoff A, Kremer P, Benesch C, Fruh K, Klank A, Kunze S: Overdrainage and shunt technology. A critical comparison of programmable, hydrostatic and variable-resistance valves and flow-reducing devices. Childs Nerv Syst 11:193-202, 1995

4. Bouzerar R, Czosnyka M, Czosnyka Z, Balédent O: Physical phantom of craniospinal hydrodynamics. Acta Neurochir Suppl 113:65-69, 2012

5. Brimioulle S, Moraine JJ, Norrenberg D, Kahn RJ: Effects of positioning and exercise on intracranial pressure in a neurosurgical intensive care unit. Phys Ther 77:1682-1689, 1997

6. Browd SR, Gottfried ON, Ragel BT, Kestle JR: Failure of cerebrospinal fluid shunts: part II: overdrainage, loculation, and abdominal complications. Pediatr Neurol 34:171-176, 2006

7. Browd SR, Ragel BT, Gottfried ON, Kestle JR: Failure of cerebrospinal fluid shunts: part I: Obstruction and mechanical failure. Pediatr Neurol 34:83-92, 2006

8. Chapman PH, Cosman ER, Arnold MA: The relationship between ventricular fluid pressure and body position in normal subjects and subjects with shunts: a telemetric study. Neurosurgery 26:181-189, 1990

9. Chari A, Czosnyka M, Richards HK, Pickard JD, Czosnyka $\mathrm{ZH}$ : Hydrocephalus shunt technology: 20 years of experience from the Cambridge Shunt Evaluation Laboratory. J Neurosurg 120:697-707, 2014

10. Coté GL, Durai R, Zoghi B: Nonlinear closed-loop control system for intracranial pressure regulation. Ann Biomed Eng 23:760-771, 1995

11. Czosnyka M, Czosnyka Z, Momjian S, Pickard JD: Cerebrospinal fluid dynamics. Physiol Meas 25:R51-R76, 2004

12. Czosnyka M, Czosnyka Z, Whitehouse H, Pickard JD: Hydrodynamic properties of hydrocephalus shunts: United Kingdom Shunt Evaluation Laboratory. J Neurol Neurosurg Psychiatry 62:43-50, 1997

13. Czosnyka Z, Czosnyka M, Richards H, Pickard JD: Hydrodynamic properties of hydrocephalus shunts. Acta Neurochir Suppl 71:334-339, 1998

14. Czosnyka Z, Czosnyka M, Richards HK, Pickard JD: Laboratory testing of hydrocephalus shunts-conclusion of the U.K. Shunt evaluation programme. Acta Neurochir (Wien) 144:525-538, 2002

15. Czosnyka ZH, Cieslicki K, Czosnyka M, Pickard JD: Hydrocephalus shunts and waves of intracranial pressure. Med Biol Eng Comput 43:71-77, 2005

16. Daley ML, Gallo AE, Gehling GF, Isom JB, Mauch W, Kingsley PD: Fluctuation of intracranial pressure associated with the cardiac cycle. Neurosurgery 11:617-621, 1982

17. Drake JM, Kestle JR, Tuli S: CSF shunts 50 years on-past, present and future. Childs Nerv Syst 16:800-804, 2000

18. Dunn LT: Raised intracranial pressure. J Neurol Neurosurg Psychiatry 73 (Suppl 1):i23-i27, 2002

19. Elixmann IM, Walter M, Kiefer M, Leonhardt S: Simulation of existing and future electromechanical shunt valves in combination with a model for brain fluid dynamics. Acta Neurochir Suppl 113:77-81, 2012

20. Foltz EL, Blanks J, Meyer R: Hydrocephalus: the zero ICP ventricle shunt (ZIPS) to control gravity shunt flow. A clinical study in 56 patients. Childs Nerv Syst 10:43-48, 1994

21. Fox JL, McCullough DC, Green RC: Effect of cerebrospinal fluid shunts on intracranial pressure and on cerebrospinal fluid dynamics. 2. A new technique of pressure measurements: results and concepts. 3. A concept of hydrocephalus. J Neurol Neurosurg Psychiatry 36:302-312, 1973

22. Juul N, Morris GF, Marshall SB, Marshall LF: Intracranial hypertension and cerebral perfusion pressure: influence on neurological deterioration and outcome in severe head injury. J Neurosurg 92:1-6, 2000

23. Kasprowicz M, Asgari S, Bergsneider M, Czosnyka M, Hamilton R, Hu X: Pattern recognition of overnight intracranial pressure slow waves using morphological features of intracranial pressure pulse. J Neurosci Methods 190:310-318, 2010

24. Keong N, Czosnyka M, Czosnyka Z, Pickard JD: Clinical evaluation of adult hydrocephalus, in Winn H (ed): Youman's Neurological Surgery, ed 6. Philadelphia: Elsevier, 2011

25. Khasnis A, Lokhandwala Y: Clinical signs in medicine: pulsus paradoxus. J Postgrad Med 48:46-49, 2002

26. Ko WH, Meyrick CW, Rekate HL: Cerebrospinal fluid control system. Proc IEEE 76:1226-1235, 1988

27. Krause I, Hahne S, Walter M, Kiefer M, Linke S, Radermacher K, et al: Brain pressure dynamics and control with external ventricular drainage, in Jobbágy Á (ed): 5th European Conference of the International Federation of Medical 
and Biological Engineering. Berlin: Springer, 2012, Vol 37, pp 315-318

28. Lemaire JJ, Khalil T, Cervenansky F, Gindre G, Boire JY, Bazin JE, et al: Slow pressure waves in the cranial enclosure. Acta Neurochir (Wien) 144:243-254, 2002

29. Lundkvist B, Eklund A, Kristensen B, Fagerlund M, Koskinen LO, Malm J: Cerebrospinal fluid hydrodynamics after placement of a shunt with an antisiphon device: a long-term study. J Neurosurg 94:750-756, 2001

30. Lutz BR, Venkataraman P, Browd SR: New and improved ways to treat hydrocephalus: Pursuit of a smart shunt. Surg Neurol Int 4 (Suppl 1):S38-S50, 2013

31. Magnaes B: Body position and cerebrospinal fluid pressure. Part 1: clinical studies on the effect of rapid postural changes. J Neurosurg 44:687-697, 1976

32. Magnaes B: Body position and cerebrospinal fluid pressure. Part 2: clinical studies on orthostatic pressure and the hydrostatic indifferent point. J Neurosurg 44:698-705, 1976

33. Magnaes B: Movement of cerebrospinal fluid within the craniospinal space when sitting up and lying down. Surg Neurol 10:45-49, 1978

34. Mokri B: The Monro-Kellie hypothesis: applications in CSF volume depletion. Neurology 56:1746-1748, 2001

35. Momani L, Al-Nuaimy W, Al-Jumaily M, Mallucci C: A mechatronic valve in the management of hydrocephalus: methods and performance. Med Biol Eng Comput 49:121-132, 2011

36. Momani L, Alkharabsheh AR, Al-Nuaimy W: Design of an intelligent and personalised shunting system for hydrocephalus. Conf Proc IEEE Eng Med Biol Soc 2008:779-782, 2008

37. Momani L, Alkharabsheh AR, Al-Zuibi N, Al-Nuaimy W: Instantiating a mechatronic valve schedule for a hydrocephalus shunt. Conf Proc IEEE Eng Med Biol Soc 2009:749_ 752,2009

38. Nulsen FE, Spitz EB: Treatment of hydrocephalus by direct shunt from ventricle to jugular vain. Surg Forum 1951:399403, 1951

39. Sakka L, Coll G, Chazal J: Anatomy and physiology of cerebrospinal fluid. Eur Ann Otorhinolaryngol Head Neck Dis 128:309-316, 2011

40. Wåhlin A, Ambarki K, Birgander R, Alperin N, Malm J, Eklund A: Assessment of craniospinal pressure-volume indices. AJNR Am J Neuroradiol 31:1645-1650, 2010

\section{Disclosures}

Drs. Browd and Lutz report that they own stock in Aqueduct Neurosciences Inc. and Aqueduct Critical Care Inc.

\section{Author Contributions}

Conception and design: Lutz. Acquisition of data: Venkataraman. Analysis and interpretation of data: Venkataraman. Drafting the article: Venkataraman. Critically revising the article: Lutz, Browd. Reviewed submitted version of manuscript: all authors Approved the final version of the manuscript on behalf of all authors: Lutz. Administrative/technical/material support: Browd. Study supervision: Lutz.

\section{Supplemental Information}

Online-Only Content

Supplemental material is available with the online version of the article.

Supplementary Figures. http://thejns.org/doi/suppl/10.3171/ 2016.2.PEDS15478

\section{Correspondence}

Barry R. Lutz, Department of Bioengineering, University of Washington, 3720 15th Ave. NE, Seattle, WA 98195-5061. email: blutz@uw.edu. 